\title{
Desarrollo embrionario-larval y tiempo de metamorfosis del pez tropical Xenomelaniris brasiliensis (Pisces: Atherinidae)
}

\author{
Veronica del Río ${ }^{1}$, Jesús Rosas ${ }^{2}$, Aidé Velásquez ${ }^{1} \&$ Tomas Cabrera $^{1}$ \\ 1 Escuela de Ciencias Aplicadas del Mar. \\ 2 Instituto de Investigaciones Científicas. Universidad de Oriente Núcleo de Nueva Esparta, Boca del Río, estado Nueva \\ Esparta, Venezuela. Telefax 02952911350. rosas@ne.udo.edu.ve
}

\author{
Recibido 29-IV-2005. Corregido 06-VII-2005. Aceptado 10-VIII-2005.
}

\begin{abstract}
Development of the tropical fish Xenomelaniris brasiliensis (Pisces: Atherinidae) from embryo to larva and time to metamorphosis. Embryonic-larval development, and metamorphosis larval time, were studied in the tropical fish Xenomelaniris brasiliensis. Twenty nine sexually mature specimens were used, 16 females $(10.86 \pm 1.01 \mathrm{~cm}$ and $7.63 \pm 2.62 \mathrm{~g})$ and 13 males $(10.43 \pm 0.57 \mathrm{~cm}$ and $6.54 \pm 1.44 \mathrm{~g})$ which produced gametes through abdominal massage. Fertilized eggs were spherical (1.18 $\pm 0.44 \mathrm{~mm}$ diameter), greenish, transparent, benthonic and vitelus-rich; rugose striated chorion with numerous external filaments randomly distributed and abundant oil globules $\left(0.11 \pm 0.07 \mathrm{~mm}\right.$ diameter). The embryonic development was finished at $26.36 \pm 2.03^{\circ} \mathrm{C}$, $39.67 \pm 0.58 \mathrm{PSU}$ and $\mathrm{pH} 8.30 \pm 0.10$. Larvae $(4.56 \pm 0.97 \mathrm{~mm}$ total length) hatched at 143 hours and 19 minutes, with vitteline sac vestiges and a single oil globule. The larvae were fed on Brachionus plicatilis and Isochrysis galbana. After the second week Artemia nauplii were added and I. galbana maintained. Flexion started 13 days after larvae hatched $(6.10 \pm 1.54 \mathrm{~mm}$ total length) and was completed 32 days later (11.25 $\pm 1.87 \mathrm{~mm}$ total length) with the hipural complex completely developed. In conclusion, $X$. brasiliensis showed direct larval development and started larval metamorphosis (13.08 $\pm 2.07 \mathrm{~mm}$ total length) to juvenile 40 days after hatching. Rev. Biol. Trop. 53(3-4): 503-513. Epub 2005 Oct 3.
\end{abstract}

Key words: Embryonic development, metamorphosis, Xenomelaniris brasiliensis.

El tinicalo Xenomelaniris brasiliensis (Quoy y Gaimard, 1824) pertenece a la familia Atherinidae y abundan en aguas protegidas e interior de las lagunas litorales salobres o hipersalinas. Se distribuye desde el Mar Caribe en Venezuela hasta el sureste de Brasil (Cervigón 1991). Por su abundancia constituye un importante eslabón en la trama trófica del ecosistema pelágico costero, se emplea en la pesca artesanal como carnada (Randall 1977, Marín et al. 1995, Allen et al. 2003). Sin embargo, existe escasa información bibliográfica y tan sólo destacan trabajos sobre su alimentación Carvalho (1953a), aspectos parasitarios (Carvalho 1953b, 1955a, 1955b), descripción de algunos aspectos biológicos (Carreño 1975) y descripción de los ovocitos y postlarvas (Marín et al. 1995). Los objetivos del presente estudio fueron describir el desarrollo embrionario y larval de $X$. brasiliensis y determinar el tiempo de metamorfosis, dada la importancia ecológica de la especie.

\section{MATERIALES Y MÉTODOS}

Los ejemplares adultos (16 hembras de $10.86 \pm 1.01 \mathrm{~cm}$ y $7.63 \pm 2.62 \mathrm{~g}$ y 13 machos de $10.43 \pm 0.57 \mathrm{~cm}$ y $6.54 \pm 1.44 \mathrm{~g}$ ) fueron capturados en aguas adyacentes a la población de Boca de Río, Isla de Margarita, Venezuela $\left(10^{\circ} 95^{\prime} \mathrm{N}, 64^{\circ} 27^{\prime} \mathrm{W}\right)$ y trasladados al laboratorio. Mediante un suave masaje abdominal (Silva 1995) expulsaron sus productos sexuales; los ovocitos maduros y el líquido espermático fueron recolectados en recipientes de 31 . La fertilización se realizó en seco (Woynarovich 1986) con un $80 \pm 4 \%$ de fertilización. Los 
huevos fértiles se observaron cada hora con el fin de determinar los estadios y el tiempo hasta la eclosión de las larvas según lo propuesto por Balinsky (1978). El criterio de descripción larval se realizó según Ahlstrom (1968). Las fotografías se hicieron con cámara digital y microscopio. Simultáneamente se registraron la temperatura y la salinidad con un termómetro de $1^{\circ} \mathrm{C}$ y un refractómetro de 0.5 PSU de precisión respectivamente.

En cinco recipientes circulares de 501 provistos de aireación continua se colocaron 80 larvas, suministrando como alimento el rotífero Brachionus plicatilis (7 rot $/ \mathrm{ml}$ ) y microalgas (Isochrysis galbana) a $50000 \mathrm{cel} / \mathrm{ml}$ durante 13 días, y finalmente nauplios de Artemia. Cada tres días se midieron 5 larvas fijadas con formalina al $5 \%$ para determinar los parámetros morfológicos (longitud total, longitud notocordial, longitud postanal, ancho del cuerpo, diámetro ocular) hasta completar la metamorfosis (Silva 1995).

\section{RESULTADOS}

Descripción de los ovocitos: Los huevos inviables midieron $1.05 \pm 0.30 \mathrm{~mm}$ con filamentos externos opacos con gotas lipídicas internas (Fig. 1A), los huevos fértiles fueron esféricos, verdosos, translúcidos, bentónicos, telolecitos, ricos en vitelo, adhesivos y con un corion rugoso y estriado, con un diámetro de $1.18 \pm 0.44 \mathrm{~mm}$ (Fig. 1B).

Todos los huevos presentaron internamente entre $6.5 \pm 3.04$ gotas de aceite por ovocito. $\mathrm{Su}$ mayor diámetro fue de $0.19 \pm 0.06 \mathrm{~mm}$ y el menor de $0.07 \pm 0.02 \mathrm{~mm}$. Los fértiles formaron agregaciones debido a la presencia de filamentos coriónicos no ramificados y distribuidos aleatoriamente por toda la superficie externa (Fig. 1C). Se contabilizaron $5.77 \pm 1.79$ filamentos por ovocitos, con un leve giro sobre sí mismo y con un punto o base de inserción en el corion. Estas bases midieron $0.04 \pm 00 \mathrm{~mm}$ de altura y $0.04 \pm 00 \mathrm{~mm}$ de ancho.

En los huevos se puedo observar el proceso de activación debido al desenvolvimiento de los filamentos coriónicos a medida que se propaga la exocitosis de los alvéolos corticales produciéndose la fertilización al entrar en contacto con el espermatozoide (Fig. 1D). Esto fue al contrario de los huevos no activados que se encuentran envueltos en estos filamentos. Esto ocurre incluso antes de que el espermatozoide penetre en el interior del óvulo. La "ola" de exocitosis se propaga sobre la superficie completa del huevo desde la zona adyacente al micrópilo y finaliza en el polo vegetativo, originándose una separación entre el ooplasma y la membrana coriónica la cual se endurece y se vuelve más fina, formándose el espacio previtelino.

Fertilización: Desde la fertilización del huevo hasta la eclosión de las larvas, la temperatura fue de $26.36 \pm 2.03^{\circ} \mathrm{C}$, la salinidad 39.67 \pm 0.58 PSU y el pH $8.30 \pm 0.10$. No hubo crecimiento en el tamaño de los huevos, de los filamentos coriónicos o de las gotas lipídicas una vez que coalescen. La fertilización se produjo cuando el cono de fecundación engloba gradualmente al espermatozoide y luego empieza a retraerse, transportando al espermatozoide hacia adentro.

Etapas de "clivaje": La segmentación se inició con la división del núcleo (1.18hrs), a la cual siguió la división del citoplasma, contracciones oscilatorias causaron la migración del citoplasma cortical periférico hacia el polo animal, donde se formó el blastodisco. Dos pequeñas marcas en el blastodisco sirven para identificar la localización del surco de segmentación. El plano de esta primera división fue generalmente vertical. La célula se dividió en dos células hijas (Fig. 1E), denominadas blastómeros (1.45 hrs), (Fig. 1F). Las gotas de aceite migran hacia el polo vegetativo y coalescen aumentando de tamaño. El plano de la segunda división fue vertical y atravesó al eje principal pero formó ángulos rectos con el primer plano de segmentación. El plano de la tercera división (2.00 hrs) formó ángulos rectos con los dos primeros planos y el eje principal del huevo (Fig. 1G). De los ocho blastómeros, cuatro se 

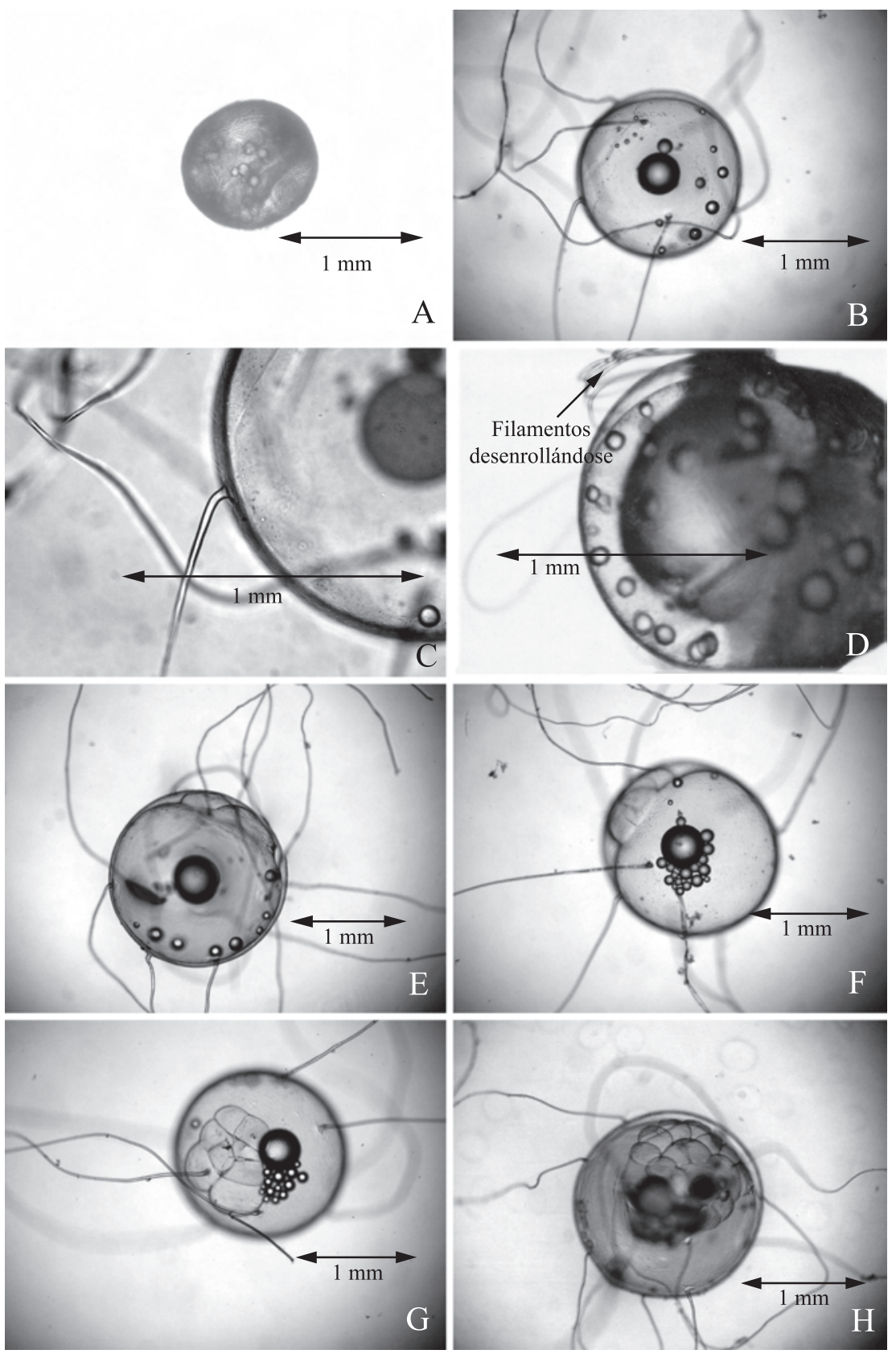

Fig. 1. Desarrollo embrionario de Xenomelaniris brasiliensis. A) Huevo infértil, B) Huevo fértil, C) Inserción de filamento coriónico, D) Liberación de los filamentos coriónicos, E) Primera división, F) Segunda división, G) Tercera división, H) Cuarta división.

Fig. 1. Embryonic development of $X$. brasiliensis. A) Unviable egg, B) Viable egg, C) Chorionic filament insertion, D) Chorionic filament liberation, E) First cleavage, F) Second cleavage, G) Third cleavage, H) Fourth cleavage. 
situaron encima de los otros cuatro, igualmente superpuestos. Los cuatro primeros blastómeros incluyendo el hemisferio animal del huevo y los restantes en el hemisferio vegetativo. El plano de la cuarta división celular (2.33 hrs) en este caso fue paralelo al de la segunda división y dividió las dos filas de blastómeros en cuatro blastómeros cada una, formándose 16 blastómeros (Fig 1H). La quinta división celular $(2.55 \mathrm{hrs})$ dio origen a 32 blastómeros (Fig. 2A).

Durante la sexta división celular (5.25 hrs) el embrión adquiere el aspecto característico de una mora, por lo cual es denominado mórula (Fig 2B), observándose hacia el polo animal del huevo. Los blastómeros se dispusieron en una capa, y se originó la cavidad llamada blastocele dispuesta internamente entre la capa germinal y el vitelo. A medida que la segmentación progresó, la capa epitelial de células englobó al blastocele, y el embrión se convirtió en una esfera hueca, cuyas paredes estaban formadas por una capa epitelial de células denominada blastodermo, formándose la blástula (Fig 2C). Las células del blastodermo fueron más pequeñas que en las etapas previas, formando capas de cuatro o cinco filas de células, lo que dificultó el conteo de las células de la siguiente división.

Gastrulación, neurulación y desarrollo del embrión: Al inicio del proceso de gastrulación (18.55 hrs) (Fig. 2D), el blastodermo se expandió sobre la superficie del vitelo por epibolia, caracterizada por un crecimiento y multiplicación rapida de las células que formaron el blastodisco sin que hubiera un aumento apreciable en su masa. Las células empezaron a extenderse superficialmente sobre la zona vegetativa del huevo, englobándolo. A consecuencia de ello el blastodisco cubrió el vitelo, hasta que sus bordes convergieron y se cerraron en el extremo posterior al embrión formando el tampón vitelino (Fig. 2E) con lo cual concluyó la gastrulación, producto del coalescimiento de las gotas de aceite se observó sólo una en este estadio.
El proceso de neurulación (23.55 hrs) se caracterizó por la formación del escudo embrionario, como una delgada línea segmentada en el blastodermo, se reconoció la formación de la cabeza rudimentaria y se distinguió el cuerpo embrionario (Fig. 2F) una masa de células similar a un pico frente a la cabeza y las vesículas ópticas rudimentarias apreciaron a cada lado del extremo cefálico (Fig. 2F). La vesícula de Kupffer se observó en la posición extrema caudal (Fig. 2G), seguidamente se observó los esbozos primarios del corazón (Fig. 2H) protocerebro, notocordio (Fig. 3A), tubo neural y los somitos de los cuales se contabilizaron nueve (09) de $0.34 \pm 0.06 \mathrm{~mm}$ de longitud (Fig. 3 B). Se observó una sola gota de aceite relativamente grande $(0.30 \pm 0.08 \mathrm{~mm})$ dispuesta entre la cabeza y la cola del embrión a una distancia de $0.25 \pm 0.03 \mathrm{~mm}$ de la cabeza, formada por reabsorción de todas las gotas lipídicas presentes dentro del vitelo antes de empezar el proceso de desarrollo embrionario (Marín et al. 1995). Los ojos bien desarrollados midieron $0.16 \pm 0.02 \mathrm{~mm}$, el ancho de la región cefálica $0.27 \pm 0.02 \mathrm{~mm}$ y el ancho del notocordio $0.11 \pm 0.06 \mathrm{~mm}$.

El embrión presentó un desarrollo más definido (Fig. 3C), aumentó de tamaño, ocupando $50 \%$ del volumen de huevo. Se observaron los lentes ópticos, las cápsulas óticas y los latidos del corazón (107.5 \pm 7.78 por minuto). El embrión realizó pequeñas contracciones, aproximadamente 3 por minuto. Se observaron melanóforos en la zona ventral, los cuales se encontraban más agregados hacia la zona anterior y dispersos hacia la zona posterior. El vitelo se contrajo en la zona donde se encontraba el embrión, desplegándose el vitelo de la membrana coriónica. Se contabilizaron 18 somitos y se observó el canal de circulación vitelino (Fig. 3D), anteriormente reportado por Sprague et al. (2001). A las 53 hr: 07 min, se observó completamente definida la circulación del fluido en los canales vitelinos. La distancia entre la cabeza del embrión y la gota de aceite fue de $0.11 \pm 0.09 \mathrm{~mm}$. Se apreciaron los esbozos de los otolitos. 

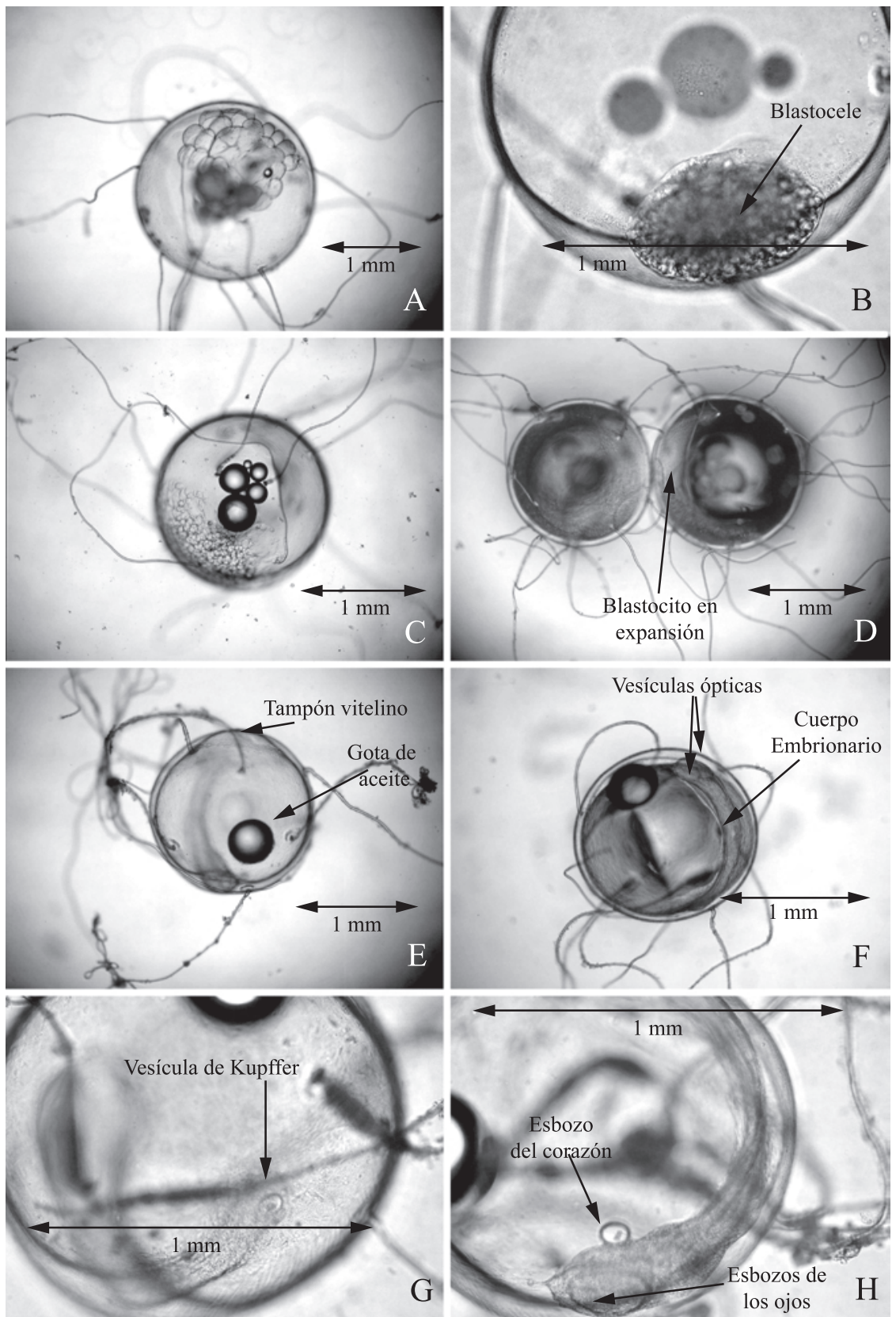

Fig. 2. Desarrollo embrionario de Xenomelaniris brasiliensis. A) Quinta división celular, B) Mórula, C) Blástula, D) Gástrula, E) Néurula temprana, F) Formación del escudo embrionario, G) Néurula con vesícula de Kupffer, H) Esbozos primarios del corazón y los ojos.

Fig. 2. Embryonic development of $X$. brasiliensis. A) Fifth cleavage, B) Morula stage, C) Blastula stage, D) Gastrula stage, E) Early neurula, F) Embryo shield formation, G) Neurula with Kupffer vesicle, H) Heart and eyes primaries. 

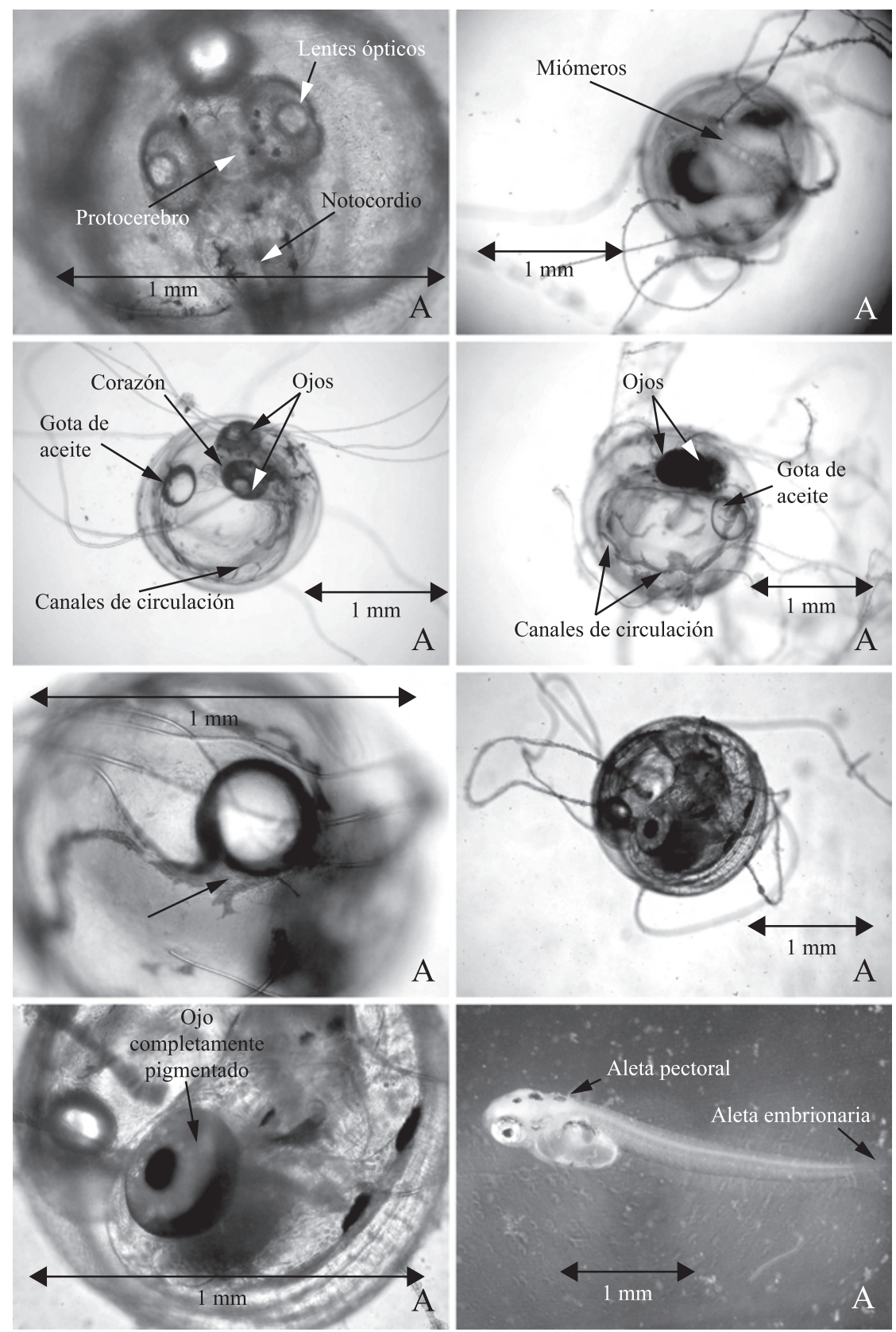

Fig. 3. Desarrollo embrionario de Xenomelaniris brasiliensis. A) Esbozos primarios de lentes ópticos, protocerebro y notocordio, B) Miómeros, C) Embrión de X. brasiliensis, D) Canales de circulación vitelina, E) Engrosamiento de los canales de circulación, F) Embrión con ojos pigmentados, G) Detalle de los ojos, H) Larva recién eclosionada.

Fig. 3. Embryonic development of Xenomelaniris brasiliensis. A) Optical capsules, brain and notochord primaries, B) Myomeres, C) Embryo stage, D) Vitteline circulation channels, E) Circulation channels thickness, F) pigmentation eyes of embryo, G) eyes details, H) Recently hatch larva. 
A las 70 hrs: 38 min, el embrión ocupó un $60 \%$ del volumen del huevo con canales de circulación vitelinos engrosados (Fig 3E), presentando acumulación de lo que pareció ser materia orgánica. Se observó un nuevo canal de circulación. El corazón midió $0.15 \pm 0.06 \mathrm{~mm}$, el embrión extendido presentó una longitud total de $1.90 \pm 0.43 \mathrm{~mm}$, con diámetro ocular de $0.30 \pm 0.04 \mathrm{~mm}$, el patrón de pigmentación del embrión presentó en la zona cefálica melanóforos pardos o negros dendriformes, formando un círculo dorsalmente en la cabeza y algunos blanquecinos dispersos en el cuerpo. Transcurridas 74 hrs: 10 min se observó los esbozos de las aletas pectorales, la longitud total del embrión extendido fue de $2.72 \pm 0.51 \mathrm{~mm}$.

A las $96.30 \mathrm{hrs}$, la longitud total fue de $3.73 \pm 0.33 \mathrm{~mm}$. El corazón desarrollado se contabilizan $194 \pm 9.17$ latidos por minuto. El diámetro de los ojos completamente pigmentados fue de $0.40 \pm 0.05 \mathrm{~mm}$. A las $117 \mathrm{hrs}$ : $16 \mathrm{~min}$, el vitelo se redujo un $80 \%$ y el embrión se observó retorcido dentro del huevo quedando superpuesto sobre sí mismo. Se detalló esbozos de la abertura bucal. Se apreció movimientos en un plano moderado de los ojos. Las aletas pectorales presentaron contracciones rápidas similares a aleteos, hasta de $20.75 \pm 6.65$ por minuto. A las 143 hrs: 50 min se observó la eclosión de las larvas a $26.36 \pm 2.03^{\circ} \mathrm{C}$ y $39.67 \pm 0.58 \mathrm{PSU}$. Los ojos totalmente pigmentados (Fig. F, G) determinó le inicio de la alimentación exógena, coincidiendo con el momento en que el saco vitelino fue consumido casi completamente lo que se denominó como "período crítico".

El porcentaje de eclosión fue de $36.03 \pm$ $8.96 \%$. Las larvas presentaron longitud total de $4.56 \pm 0.97 \mathrm{~mm}$, longitud notocordal de 4.07 $\pm 0.82 \mathrm{~mm}$, ancho del cuerpo (medida en su parte más ancha) de $0.60 \pm 0.02 \mathrm{~mm}$, diámetro ocular de $0.36 \pm 0.05 \mathrm{~mm}$, longitud de las aletas pectorales de $0.35 \pm 0.03 \mathrm{~mm}$, presentando un lóbulo de $0.15 \pm 0.01 \mathrm{~mm}$. (Cuadro 1). No se observó la aleta caudal bien desarrollada aunque si presentó esbozos de ésta y de la aleta dorsal (Fig. 3H).

Las larvas recién eclosionadas realizaron movimientos natatorios rápidos, cortos y definidos. La longitud post-anal no pudo ser determinada debido a que la apertura de la cloaca no fue definida pero si el intestino. La gota lipídica se ubicó internamente y midió $0.22 \pm 0.02 \mathrm{~mm}$. Se apreciaron tres arcos branquiales, nueve melanóforos en la zona cefálica dorsal, una línea de melanóforos dorsal y una ventral.

CUADRO 1

Valores promedio ( \pm desviación estándar) de características anatómicas de cinco larvas de Xenomelaniris brasiliensis desde la eclosión hasta la metamorfosis

TABLE 1

Average values $( \pm S D)$ of anatomic characteristics of Xenomelaniris brasiliensis five larvae from hatch to metamorphosis

\begin{tabular}{|c|c|c|c|c|c|c|}
\hline$(\mathrm{mm})$ & 1 día & 2 días & 13 días & 16 días & 32 días & 40 días \\
\hline LT & $5.04 \pm 1.22$ & $4.96 \pm 0.86$ & $6.10 \pm 1.54$ & $7.08 \pm 1.54$ & $11.25 \pm 1.87$ & $13.08 \pm 2.07$ \\
\hline $\mathrm{LN}$ & $3.94 \pm 0.04$ & $4.35 \pm 0.54$ & $5.53 \pm 0.62$ & $6.32 \pm 0.42$ & $9.30 \pm 0.75$ & $10.73 \pm 0.70$ \\
\hline LAP & $1.60 \pm 0.15$ & $1.46 \pm 0.02$ & $1.91 \pm 0.02$ & $2.32 \pm 0.04$ & $4.28 \pm 0.02$ & $5.16 \pm 0.02$ \\
\hline A & $0.63 \pm 0.03$ & $0.65 \pm 0.01$ & $0.78 \pm 0.11$ & $0.97 \pm 0.10$ & $1.37 \pm 0.01$ & $1.80 \pm 0.01$ \\
\hline LAL & $0.46 \pm 0.13$ & $0.49 \pm 0.01$ & $0.62 \pm 0.01$ & $0.83 \pm 0.08$ & $1.32 \pm 0.01$ & $1.86 \pm 0.02$ \\
\hline LL & $0.19 \pm 0.01$ & $0.21 \pm 0.01$ & $0.26 \pm 0.02$ & $0.29 \pm 0.01$ & $0.41 \pm 0.04$ & $0.74 \pm 0.02$ \\
\hline DO & $0.40 \pm 0.10$ & $0.39 \pm 0.02$ & $0.50 \pm 0.22$ & $0.58 \pm 0.01$ & $0.54 \pm 0.02$ & $1.11 \pm 0.10$ \\
\hline
\end{tabular}

LT: Longitud Total, LN: Longitud Notocordal, LAP: Longitud Post-Anal, A: Ancho del cuerpo, LAL: Longitud de las Aletas pectorales, LL: Longitud del Lóbulo de las aletas pectorales, DO: Diámetro Ocular. 
Desarrollo larval y tiempo de metamorfosis: Las larvas de 24 horas de edad de 5.04 $\pm 1.22 \mathrm{~mm}$ de longitud total (Lt), presentaron el intestino completamente desarrollado y la capacidad de perseguir a sus presas, la apertura de la boca midió $0.321 \pm 0.08 \mathrm{~mm}$ de alto e internamente sólo se observaron vestigios del saco vitelino.

Luego de 48 horas las larvas midieron $4.96 \pm 0.86 \mathrm{~mm}$ de Lt, apreciándose los arcos branquiales, los opérculos definidos y la osificación de sus estructuras. El día 13 la Lt la fue $6.10 \pm 1.54 \mathrm{~mm}$, iniciándose la flexión, proceso que implica la curvatura del extremo distal del notocordio para conformar el complejo hipural. El día 16 la Lt fue $7.08 \pm 1.54 \mathrm{~mm}$ y comenzó la dentición, observándose 4 dientes en la mandíbula. El proceso de flexión concluyó a los 32 días luego de la eclosión, la Lt fue de 11.25 $\pm 1.87 \mathrm{~mm}$, detallándose el complejo hipural completamente desarrollado.

A los 40 días las larvas tenían una Lt de $13.08 \pm 2.07 \mathrm{~mm}$, iniciándose el proceso de escamación en la región mandibular y opercular, se inició la metamorfosis de larva a juvenil.

A los 40 días de edad los ejemplares de $X$. brasiliensis presentan 38 vértebras. Otro carácter distintivo en larvas es el patrón de pigmentación, en ejemplares de $14.1 \mathrm{~mm}$ de Lt. Observándose pigmentos lineales (en forma de guiones) que se extienden desde la base de la aleta pectoral a través de toda la línea lateral hasta el complejo hipural. Se observó pigmentos puntiformes a lo largo del borde ventral y dorsal, los cuales se hicieron estelados hacia el tronco y se transformaron en seis pigmentos redondeados, tres mayores y tres de menor tamaño, todos definidos sobre el cerebro; pigmentos estelados difusos sobre toda la zona bucal, pigmentos difusos en la zona opercular y pigmentos en la base de la aleta pectoral.

\section{DISCUSIÓN}

Los peces sexualmente maduros estuvieron en el ámbito referido por Favaro et al. (2003): entre 7.61 y $6.92 \mathrm{~cm}$ para hembras y machos respectivamente. Por su parte Carreño (1975) señaló como talla mínima $7.8 \mathrm{~cm}$ para las hembras maduras.

Las características y el diámetro de los huevos inviables presentados por $X$. brasiliensis coincidieron con las mencionados por Marín et al. (1995) no así con el número de gotas de aceite y el tamaño. Esta variación esta relacionada con la temperatura y la maduración de los ovocitos, durante el tiempo transcurrido desde la ovulación y a las diferencias entre ejemplares reproductores de una misma especie (Sprague et al. 2001). Al respecto White et al. (1983) refieren que pueden encontrarse numerosas gotas de aceite en el vitelo de la mayoría de las especies de Atheriniformes. En este estudio se observó, que durante el desarrollo embrionario las gotas de aceite se agregaron en el polo vegetal y se juntaron en una sola gota en el área cercana al corazón de la larva antes de la eclosión.

En el caso de los huevos fértiles formaron agregados debido a los filamentos (Carreño 1975 y Marín et al. 1995), una característica resaltante en los huevos de casi todas las especies de Atheriniformes, pudiendo variar en número y distribución en la superficie del huevo, manteniéndolos unidos entre ellos o al substrato (White et al. 1983). Marín et al. (1995) refieren la existencia de 9 filamentos coriónicos distribuidos únicamente en el polo vegetal, con proyecciones coriónicas en la base de éstos y sin ramificaciones. White et al. (1983) señalan que aparentemente no existe ningún patrón en cuanto al número y distribución de estos filamentos pero hasta que exista mayor información disponible a este respecto, será difícil determinar la importancia filogenética de esta variabilidad en número, tamaño y ubicación en los huevos de la familia Atheriniforme.

En los Atheriniformes la fecundación es monoespermia. Zanuy (1975), Balinsky (1978) y Blaxter (1988) destacan a la reacción cortical la cual puede actuar como defensa, puesto que los mucopolisacáridos contenidos en los alvéolos corticales se liberan en el espacio previtelino, impidiendo la penetración de 
espermatozoides adicionales una vez que el primer ha establecido contacto con el óvulo. Otro factor, es que el canal micropilar presenta un diámetro que sólo permite el paso de un espermatozoide, o bien éste se hace demasiado estrecho una vez fecundado el óvulo impidiendo la penetración de otros espermatozoides (Querales et al. 2004).

El desarrollo de estos huevos es meroblástico (Balinsky 1978). Sprague et al. (2001), López et al. (2002) afirman que las divisiones consecutivas de los blastómeros no están separadas por períodos de crecimiento y los blastómeros resultantes tienen sólo la mitad del tamaño original después de cada división. Durante el proceso de desarrollo se observó la vesícula de Kupffer. Zanuy (1975) sugiere que puede tratarse de una especie de sistema hepatopancreático propio de los embriones de peces en desarrollo, que es reabsorbido durante el proceso de neurulación. Igualmente se detalló el canal de circulación vitelino. White et al. (1983), al respecto lo describe como simple y sin ramificaciones. En el embrión aparecen los esbozos de las aletas pectorales.

Los resultados referentes al tiempo de eclosión de las larvas de $X$. brasiliensis fueron explicados de acuerdo a Kamler (1992) quien señala que la calidad de la descendencia es influenciada por un efecto combinado tanto de propiedades endógenas de los reproductores, como factores internos y externos de la progenie. Para citar un ejemplo, Glamuzina et al. (1989) afirman que dentro del rango de temperatura óptima de desarrollo de Dentex dentex, cualquier elevación resulta en un aumento de la mortalidad, sin embargo los aumentos graduales de esta variable hasta un rango tolerado por la especie, mejoran su sobrevivencia. La temperatura utilizada en este estudio se encontró dentro del promedio anual $\left(24.9-31.7^{\circ} \mathrm{C}\right)$ en la laguna de La Restinga, en cuyas zonas adyacentes fueron capturados los progenitores de X. brasiliensis (Ramírez 1996).

Collins y Nelson (1993) mencionan para Siganus randalli el tiempo de eclosión entre 19.3 y 23.5 horas y la temperatura entre 27 y $30^{\circ} \mathrm{C}$. López et al. (2002) refieren un tiempo de eclosión de 17: 23 horas a $27^{\circ} \mathrm{C}$ para Diplectrum radiale, Cuartas et al. (2002) afirma que el tiempo de eclosión para Haemulom bonariense fue de $15: 19$ horas a $27^{\circ} \mathrm{C}$. Todas las especies mencionadas anteriormente al igual que la especie estudiada, son tropicales y en ninguno de estos casos los huevos sobrepasaron los $0.8 \mathrm{~mm}$ de diámetro. El diámetro de los huevos de $X$. brasiliensis fue de $1.18 \pm 0.44 \mathrm{~mm}$ a $26.36 \pm 2.03^{\circ} \mathrm{C}$, las larvas eclosionaron a las 143 horas: 50 minutos. El tiempo de desarrollo de $X$. brasiliensis en comparación con las especies mencionadas es bastante prolongado, lo cual se explica de acuerdo al tamaño de sus huevos. Silva (1995) afirma que los huevos de mayor tamaño se desarrollan más lentamente que los pequeños y que producen larvas de mayor tamaño en la eclosión, con un período más largo de alimentación sobre el vitelo.

Las larvas de la mayoría de los peces marinos, no poseen la capacidad de alimentarse inmediatamente después de su eclosión, sino que dependen del saco vitelino hasta que sus ojos y boca son funcionales. El tiempo desde la eclosión, hasta la pigmentación de los ojos representa presumiblemente el tiempo en el cual las larvas pueden nutrirse sólo de sus reservas vitelinas y no de fuentes exógenas (Houde 1974). En contraposición X. brasiliensis presentó ojos pigmentados incluso antes de la eclosión, observándose vestigios del saco vitelino lo que corroboró en este estudio los resultados obtenidos por White et al. (1983) y Marín et al. (1995), quienes afirmaron que las larvas de Atheriniformes presentan un desarrollo directo.

Algunas especies de peces eclosionan en un estado avanzado con respecto a su habilidad para alimentarse, con boca funcional y el tracto digestivo desarrollado (Kamler 1992). Durante este estudio se observó que $X$. brasiliensis posee ojos pigmentados y boca funcional al eclosionar, aunque no se observó la cloaca desarrollada. Sin embargo, el primer día después de la eclosión las larvas poseían el intestino completamente desarrollado y la capacidad efectiva de perseguir a sus presas; de acuerdo a esto se presume que las larvas de $X$. brasiliensis 
estaban en capacidad de alimentarse desde el momento de su eclosión, y no dependieron de fuentes endógenas. Esta flexibilidad en el tiempo de la primera alimentación da a las larvas una ventaja en condiciones de poca disponibilidad de alimento (Kamler 1992).

Las diferencias entre los resultados observados durante este estudio y los señalados por Marín et al. (1995) en cuanto al inicio de la escamación puede atribuirse a que los ejemplares descritos por estos autores fueron capturados del medio y preservados para su posterior estudio, en cambio los ejemplares utilizados para esta experiencia fueron cultivados y alimentados a partir de la segunda semana con nauplios de Artemia. El tipo de alimentación que presentaron las larvas durante su desarrollo y el hecho de que las larvas hallan sido preservadas para su posterior análisis pudo haber ocasionado las diferencias en los resultados.

Se concluye que el desarrollo de las larvas de $X$. brasiliensis fue directo, eclosionando con ojos completamente pigmentados, intestino bien definido sin apertura de la cloaca, con vestigios del saco vitelino y una gota lipídica interna. La flexión del notocordio se inició el día 13 concluyendo a los 32 días y la metamorfosis se inicio a los 40 días de edad.

\section{RESUMEN}

Se describe el desarrollo embrionario y larval hasta la metamorfosis de Xenomelaniris brasiliensi. Un total de 29 ejemplares sexualmente maduros, 16 hembras (10.86 \pm $1.01 \mathrm{~cm} \mathrm{y} 7.63 \pm 2.62 \mathrm{~g})$ y 13 machos $(10.43 \pm 0.57 \mathrm{~cm} \mathrm{y}$ $6.54 \pm 1.44 \mathrm{~g})$, liberaron sus productos sexuales mediante masaje abdominal. Los huevos fértiles fueron esféricos de $1.18 \pm 0.44 \mathrm{~mm}$ de diámetro, verdosos, translúcidos, bentónicos, ricos en vitelo, corion rugoso y estriado, con numerosos filamentos coriónicos, distribuidos aleatoriamente por toda la superficie externa y numerosas gotas lipídicas con un diámetro de $0.11 \pm 0.07 \mathrm{~mm}$. El desarrollo embrionario se realizó a $26.36 \pm 2.03{ }^{\circ} \mathrm{C}, 39.67 \pm 0.58 \mathrm{PSU}$ y pH $8.30 \pm 0.10$ eclosionando larvas de $4.56 \pm 0.97 \mathrm{~mm}$ a las 143 hrs: $19 \mathrm{~min}$, con vestigios del saco vitelino y una gota lipídica interna. Las larvas fueron alimentadas diariamente con Brachionus plicatilis e Isochrysis galbana, a partir de la segunda semana se incorporó nauplios de Artemia, manteniendo la adición diaria de I. galbana. A los 13 días después de la eclosión de larvas $(6.10 \pm 1.54$ mm) se inició la flexión, completándose a los 32 días $(11.25 \pm 1.87 \mathrm{~mm})$ con un complejo hipural completamente desarrollado. Se concluye que $X$. brasiliensis presentó desarrollo larval directo, iniciándose la metamorfosis de larva (13.08 \pm 2.07$)$ a juvenil a los 40 días de edad.

Palabras clave: Desarrollo embrionario, metamorfosis, Xenomelaniris brasiliensis.

\section{REFERENCIAS}

Ahlstrom, E. 1968. Review of: Development of fishes of the Chesapeake Bay Region, an atlas of egg, larval and juvenile stages. Copeia 1: 648-651.

Allen, T., R. Tavares., B. Marín., A. Barrios., E. Villarroel., M. Balza \& W. Velásquez. 2003. Determinación del crecimiento post-larvario del tinicalo Xenomelaniris brasiliensis (Quoy y Gaimard) (Pisces: Atherinidae) utilizando el análisis de sus otolitos. Mem. V Cong. Venez. de Ecol. Inst. de Zool. Trop. Porlamar, Venezuela. $12 \mathrm{p}$.

Balinsky, B.I. 1978. Introducción a la embriología. Omega. Barcelona, España. 644 p.

Balza, M., M. Gutiérrez \& B. Marín. 2001. Descripción morfológica y crecimiento en los primeros estadios larvarios de la sardina Sardinella aurita (Valenciennes, 1847) (Pisces: Clupeidae). Bol. Inst. Oceanogr. Univ. Oriente. Venezuela. 40 (1-2): 91-101.

Blaxter, J. 1988. Pattern and variety in development. In W. Hoar \& D. Randall (eds.). Fish Physiology. Parte A. Academic, Nueva York, EEUU 11: 1-58.

Carreño, R. 1975. Algunos aspectos de la biología del tinícalo, Xenomelaniris brasiliensis (Quoy \& Gaimard) (Pisces:Atherinidae), del Golfo de Cariaco, Edo. Sucre. Tesis de pregrado, Universidad de Oriente, Cumaná, Venezuela. 43 p.

Carvalho, J. 1953a. Alimentaçao de Xenomelaniris brasiliensis (Quoy y Gaimard) (Pisces: Atherinidae). Univ. R. G. Norte. Bol. Inst. Biol. Mar. 4: 127-146.

Carvalho, J. 1953b. Nota sobre Lernacenicus longiventris Wilson e sua ocurrencia em Xenomelaniris brasiliensis (Quoy y Gaimard) (Pisces: Atherinidae). Bol. Inst. Ocean. 4: 181 - 190.

Carvalho, J. 1955a. Bomolochus xenomelanirisi n. sp. parásito de peixe-rei Xenomelaniris brasiliensis (Quoy y Gaimard) (Copepoda, Cyclopodia- PiscesAtherinidae). Bol. Inst. Ocean. 6: 143-151.

Carvalho, J. 1955b. Ergasilus xenomelanirisi n. sp. parásito de peixe-rei Xenomelaniris brasiliensis 
(Quoy y Gaimard) (Copepoda, Cyclopodia- PiscesAtherinidae). Bol. Inst. Ocean. 6 (1-2): 215-224.

Cervigón, F. 1991. Los peces marinos de Venezuela. Fundación Científica Los Roques. 1: 1-238. Caracas, Venezuela.

Cuartas, A., J. Rosas., A. Velásquez \& T. Cabrera. 2003. Inducción al desove, desarrollo embrionario y larval del Corocoro rayao Haemulon bonariense Cuvier, 1830 (Pisces: Haemulidae). Rev. Mar Biol. Oceanogr. 38: 27-37.

Favaro, L., S. Lopes \& H. Spach. 2003. Reprodução do peixe-rei, Atherinella brasiliensis (Quoy \& Gaimard) (Atheriniformes: Atherinidae) em uma planicie de maré adjacente à gamboa do Baguaçu, Bahía de Paranagua, Paraná, Brasil. Rev. Bras. de Zool. 20: 501-506.

Florez, F. 1972. The effect of temperature on incubation time, growth and lethality of embryos, larvae and juveniles of the ide, Idus idus (L.) Rep. Inst. Freshwat. Res. Drottingholm. 52: 50-64.

Glamuzina, B., J. Jug-Dujakovic \& I. Katavic. 1989. Prelimirarie studies on reproduction and larval rearing of common Dentex, Dentex dentex (Linnaeus, 1758). Aquac. 77: 75-84.

Houde, E. 1973. Some recent advances and unsolved problems in the culture of marine fish larvae. Proceed. Worl. Maricult. Soc. Florida, EEUU. 83-112.

Houde, E. 1974. Effects or temperature and delayed feeding on growth and survival of larvae of three species of subtropical marine fishes. Mar. Biol. 26: 271-285.

Kamler, E. 1992. Early life history of fish: An energetics aproach. Chapman \& Hall, Cornwall, Inglaterra. $267 \mathrm{p}$.

Kokurewicz, B., M. Kowalewski \& A. Witowski. 1980. Influence of constant and variable temperatures on the embryonic development of progeny. Pol. Arch. Hydrobiol. 28: 243-256.

Lagler, K., J. Bardach \& R. Millar. 1962. Ichthyology: The study of fishes. Wiley, Nueva York, Nueva York, EEUU. 545 p.

López, P., J. Rosas., A. Velásquez., T. Cabrera \& C. Maneiro. 2002. Desarrollo embrionario y larval del bolo Diplectrum radiale Quoy y Gaimard, 1824 (Pisces: Serranidae). Rev. Biol. Mar. Ocean. 37: 127-137.

Margalef. R. 1998. Ecología. Omega. Barcelona, España. $951 \mathrm{p}$.

Marín, B., O. Díaz \& R. Briceño. 1995. Aspectos descriptivos de los ovocitos y postlarva de tinícalo, Xenomelaniris brasiliensis (Quoy y Gaimard) (Pisces: Atherinidae). Bol. Inst. Oeanogr. Univ. Oriente. Venezuela. 34: 59-68.

Querales, D., J. Rosas., A. Velásquez., T. Cabrera \& C. Maneiro. 2004. Desarrollo embrionario y larval de Paralabrax dewegeri Metzelaar, 1919 (Pisces: Serranidae). Rev. Biol. Mar. Ocean. 39: 1-11.

Ramírez, P. 1996. Lagunas costeras venezolanas. Benvente \& Martínez, Caracas, Venezuela. 275 p.

Randall, J. 1977. Atherinidae. In FAO species identification sheets for fisheries purposes. Western Central Atlantic, Fishing Area 31 (1): 273-284.

Silva, A. 1995. Apuntes curso interamericano de cultivo de peces marinos. Rosales, Santiago, Chile. 360 p.

Sokahl, R. \& F. Rohlf. 1979. Biometría: Principios y métodos estadísticos en la investigación biológica. Blume. Madrid. $832 \mathrm{p}$.

Sprague, J., E. Doerry, S. Douglas \& M. Westerfield. 2001. The Zebrafish Information Network (ZFIN): a resource for genetic, genomic and developmental research. Nucleic Acids Res. 29: 87-90.

White, H., J. Lavenberg \& C. McGowen. 1983. Atheriniformes: Development and Relationships, p. 355-362. In E. Sandknop, B. Sumida \& H. Moser. 1983. Ontogeny and systematic of fishes. An Internat. Symp. Dedicate. to the Mem. of Elbert Halvor Ahlstrom. Am. Soc. of Ichtyolog. and Herpetolog. Los Angeles, California, EE.UU.

Woynarovich, E. 1986. Tambaqui e pirapitinga. Propagacao artificial e criacao de alevinos. Programa de irrigacao - CODEVASF - Compahia de desenvolvimento do Vale de Sao Francisco, Brasilia, Brasil. 68 p.

Zanuy, S. 1975. Desarrollo del huevo y estados larvarios de cabrilla (Paracentropristis cabrilla L.). Inv. Pesc. 39: 473-489. 
